\title{
SIMULATING PLAUSIBLE MECHANISMS FOR CHANGING HEPATIC XENOBIOTIC CLEARANCE PATTERNS
}

\author{
Shahab Sheikh-Bahaei \\ Joint UCSF/UCB Graduate Group in \\ Bioengineering \\ University of California \\ Berkeley, CA 94720 USA
}

\author{
C. Anthony Hunt \\ Department of Bioengineering and Therapeutic \\ Sciences \\ University of California \\ San Francisco, CA 94143 USA
}

\begin{abstract}
No concrete, causal, mechanistic theory is available to explain how different hepatic zonation patterns of P450 isozyme levels and hepatotoxicity emerge following dosing with different compounds. We used the synthetic method of modeling and simulation to discover, explore, and experimentally challenge a concrete mechanism that shows how and why biomimetic zonation patterns emerge and change within agentbased analogues. We hypothesized that those mechanisms have counterparts in rats. Mobile objects map to compounds. One analogue is comprised of a linear sequence of 20 identical, quasi-autonomous functional units called sinusoidal segments (SSs). SSs detect and respond to compound-generated response signals and the local level of a gradient. Each SS adapts to new information with the objective of improving efficiency (lowering costs). Upon compound exposure, analogues developed a variety of patterns that were strikingly similar to those reported in the literature.
\end{abstract}

\section{INTRODUCTION}

Hepatic zonation is conspicuous periportal (near where blood enters a lobule) to perivenous (near where blood exits through central vein) attribute gradients within lobules. Our interest has been in discovering plausible mechanistic explanations for the different types of dynamic, adaptive hepatic zonation phenomena that emerge following dosing with different compounds and xenobiotics (Gebhardt, 1992; Jungermann, 1995), especially zonal differences in the metabolic clearance of xenobiotics.

Here we focus on differential sensitivity to the induction of cytochrome P450 isozymes (Oinonen and Lindros, 1998) caused by dosing rats with different xenobiotics. The P450 (also called CYP) isozymes are primarily responsible for the metabolic clearance of xenobiotics. Zonation phenomena are most often ascribed to having a multifactorial basis, in which oxygen gradients and other blood-borne signals may play prominent roles (Lindros, 1997; Christoffels et al., 1999; Jungermann and Kietzmann, 2000; Benhamouche et al., 2006; Hailfinger et al., 2006; Sekine et al., 2006; Camp and Capitano, 2007; Burke et al., 2009). Recent evidence supports the hypothesis that components of the Wnt- $\beta$-catenin pathway may play an important role. Braeuning (2009) reviews the role of several pathways including Ras-Raf-MAPK (mitogen-activated, protein kinase) and Wnt- $\beta$-catenin. However, no concrete, causal, mechanistic theory has yet been offered for how different types of hepatic zonation phenomena emerge following dosing with different compounds. Christoffels et al. (1999) hypothesized that interaction between two or more, different signal gradients is necessary to enable development of periportal-to-perivenous (P-to-P) gene expression patterns. Hailfinger et al. (2006) went further and hypothesized that control of dynamic, adaptive zonation patterns requires two opposing signals, one corresponding to a stable gradient of one or more blood-borne molecules and the other arising from responding cells within the liver, such as collocated endothelial cells. 


\section{Sheikh-Bahaei and Hunt}

We used the synthetic method of modeling and simulating biological systems (Hunt et al., 2009) to construct, explore, and experimentally challenge concrete mechanisms that show how and why biomimetic zonation patterns emerge and change within an agent-based analogue of a hepatic lobule in response to compound dosing. The modeling and simulation $(\mathrm{M} \& \mathrm{~S})$ approach used (Fig. 1A) was developed to enable construction of concrete biomimetic mechanisms that were strictly defined. The models were used to mimic wet-lab phenomena obtained under conditions that are less supportive of inductive modeling methods (Fig. 1B). The abstract mechanisms and their spatial context were flexible instantiations of mechanistic hypotheses. Their plausibility was tested experimentally.

Following cycles of model construction, evaluation and selection, and refinement, we arrived at a discrete event, discrete time device, a zonally responsive lobular analogue (ZoRLA), that maps to linear segment of hepatic lobule having P-to-P flow and a connection to extrahepatic tissue. It was comprised of 20 identical, quasi-autonomous units (agents) called sinusoidal segments (SSs). Each SS maps to a small portion of a sinusoid (Fig. 2). Each SS detected and used a local gradient signal. Mobile objects that mapped to administered compounds moved through the ZoRLA. If detected by an SS, there were two consequences. The compound could be cleared, and it could cause a response: release of mobile objects that function as potency-dependent response signals. The gradient and response signals mapped to the two signals specified by Hailfinger et al. (2006). An SS used signal information and a learning algorithm to adapt to changing conditions and update its clearance effort, which is the probability of compound clearance following detection within a simulation step.

Upon compound exposure, the ZoRLA developed a variety of periportal-to-perivenous (P-to-P) clearance effort gradients. Several gradient patterns were strikingly similar to those reported in the literature (above citations) for P450 isozymes following xenobiotic dosing (for convenience, examples are provided in Supplementary Fig. S10). Zonal patterns of clearance effort changed depending on compound potency and dose. The in silico mechanism may have counterparts in rats.

\section{METHODS}

To distinguish clearly in silico components and processes from corresponding rat counterparts, we use SMALL CAPS when referring to the former. Parameter names are italicized.
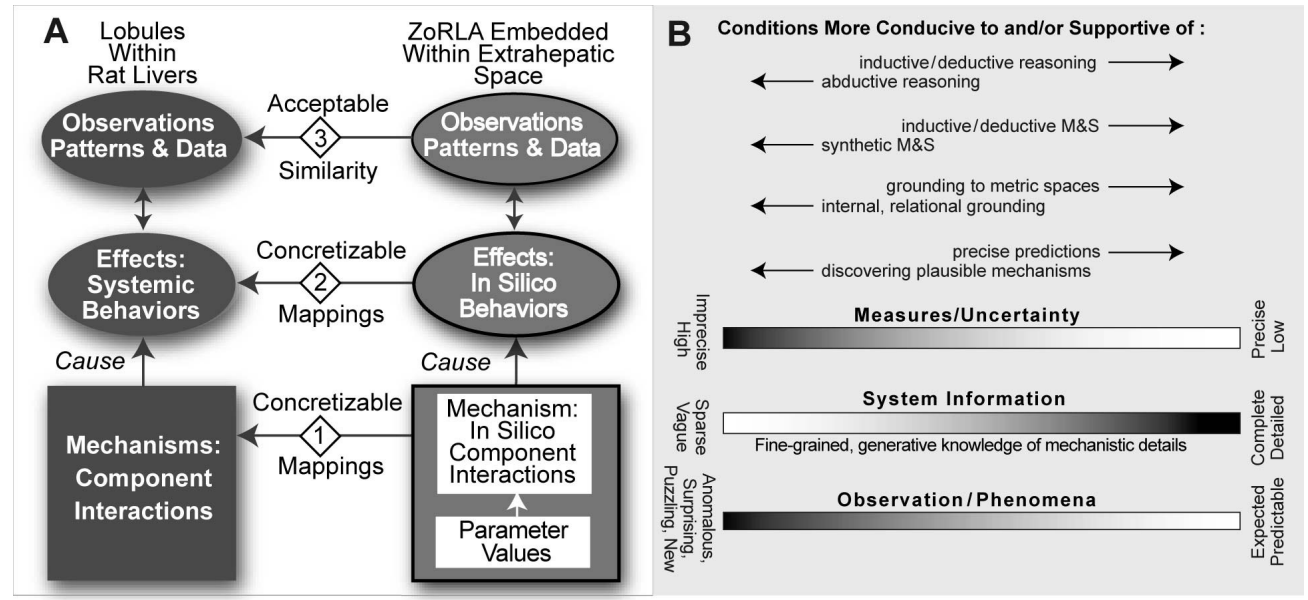

Figure 1: Features of the synthetic method of M\&S.

A: Shown are relationships between observations made on rat liver lobules following xenobiotic treatments and the ZoRLA in Fig. 2 during treatments with XENOBIOTICS. Left: the referent systems are experimental observations made on rat livers following various xenobiotic treatments. During experiments, lobular components interact with administered xenobiotics. Localized mechanistic events cause 


\section{Sheikh-Bahaei and Hunt}

systemic effects, which are reflected in the recorded data. Right: Concretizable mappings (1) exist between ZoRLA components and how they plug together, and lobular physiological and functional detail at the level of a sinusoid, as illustrated in Fig. 2. Execution gives rise to a working analogue; measures of events provide results. Measures of dynamics_-patterns of zonation, in this case-provide data that may or may not mimic wet-lab counterparts. Achieving measurable similarities makes mappings 3 quantitative. B: Sketched are conditions supportive of both the synthetic method of M\&S along with the familiar inductive method of M\&S. Conditions toward the far right side are common for non-biological, engineered systems. They favor developing inductive models that are increasingly precise and predictive. However, as discussed in (Hunt et al., 2009), absent detailed knowledge of the causes of hepatic zonation, we are on the left side, where frequent abduction is needed and synthetic M\&S methods can be most useful.

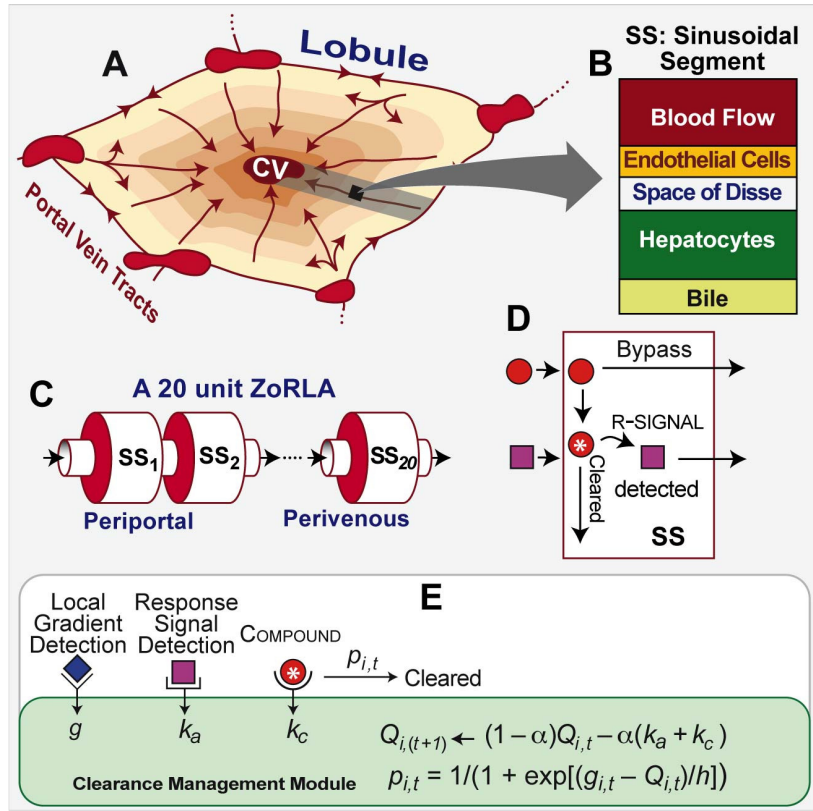

Figure 2: Referent and analogue.

A: An illustration of a cross-section through a hepatic lobule showing sinusoidal flow paths from portal vein tracts to the central vein $(\mathrm{CV})$ and a P-to-P zonation pattern. B: A Sinusoidal Segment (SS) is a quasi-autonomous agent. As illustrated here, it maps to small portion of a lobule that includes portions of the sinusoidal blood flow and space of Disse, along with one or more endothelial cells and hepatocytes. C: A ZoRLA is a connected sequence SS. To reach the CV, a COMPOUND must pass through all SS and escape being cleared. D: An SS can detect and act on mobile objects that enter. Two object types are used: COMPOUND (red) and RESPONSE SIGNALS (R-SIGNALS). Upon detection (asterisk) a COMPOUND will be cleared with probability $p_{i, t}$ (simulation step $t$ ). It may also initiate a response: create one or more mobile R-SIGNALS; the number generated is proportional to potency, $k_{t}$. E: The CLEARANCE Management Module within each SS uses algorithms as placeholders for more fine-grained intracellular mechanisms. The local value of the gradient is $g$. If a COMPOUND is undetected, it exits (bypass). When a COMPOUND is CLEARED, there is a cost, $k_{c}$. R-SIGNALS that arrive from upstream are detected. The cost of each detection event is $k_{a}$. The value of $Q$ used by $\mathrm{SS}_{i}$ during simulation step $t$ is an estimate of its long-term, discounted cost of continuing to use its current CLEARANCE effort, $p_{t}$; it is updated as specified each step using that location's $g$ value along with $Q_{i, t} . Q_{i,(t+l)}$ is the value to be used during the next step. $h$ and $\alpha$ are defined in the text. $Q$ is updated whether or not a COMPOUND and/or R-SIGNAL is detected. 


\section{Sheikh-Bahaei and Hunt}

\subsection{The Attributes Targeted}

The first consideration in any modeling effort is to determine why the models are being created, and identify situations in which they will be used. For this project, we sought spatially organized, biomimetic mechanisms that could produce phenomena similar to attributes listed in Section 2.2. Unlisted hepatic phenomena are, for the time being, outside the scope of this project. However, a requirement was that the models and their components be sufficiently flexible so that during a future extension of this project, or when other investigators use the models, they could be easily modified to account for an expanding list of attributes. Clear statements about use and targeted attributes facilitate selecting specifications. Clear specifications guide model design and development and help one avoid potentially unproductive tangents. Each attribute achieved provides a degree of validation.

To achieve targeted attributes, we followed an iterative construction and refinement protocol similar to that detailed most recently in (Hunt et al., 2009) and (Lam and Hunt, 2009). The objective was to discover CELL level mechanisms that would make ZoRLA patterns of CLEARANCE effort (defined below) following COMPOUND dosing, increasingly biomimetic, while adhering to a strong parsimony guideline. The iterative refinement protocol cycles through the following eight steps.

1) Choose an initial, small subset of attributes to target.

2) Select a granularity level that will enable comparing measures of simulated and targeted attributes. We selected the level illustrated in Fig. 2.

3) For each attribute targeted, specify a desired level of phenomenal similarity (e.g., within $\pm 25 \%$ or exhibit the same P-to-P gradient trend). Approach in stages: begin with relaxed similarity measures.

4) Posit coarse-grained, discrete mechanisms that may generate analogous phenomena while requiring as few components as is reasonable.

5) Create logic for each component. Instantiate components and mechanisms. Update specifications.

6) Conduct many, simulation experiments. Measure a variety of phenomena to establish in silico to wetlab similarity and lack thereof.

7) Tune (parameterize) to achieve analogue similarity specified at step 3 . When the effort fails, return to step 4. When successful, return to step 3 and increase the stringency of the similarity measure.

8) Add one or more new attributes until the current analogue is falsified. Return to step 2. Strive to achieve the expanded attribute list with as little component reengineering as possible.

\subsection{Targeted Attributes and Specifications}

- A (attribute): Lobules are comprised of cells, which are typically autonomous functional units. $\mathbf{S}$ (specification): Each ZoRLA is comprised of autonomous functional units called sinusoidal segments (SS) that map to a portion of a sinusoid containing one or more hepatocytes (Fig. 2).

- A: Hepatocyte function is location dependent. Cells respond based only on local information. S: An SS does the same.

- A: Compounds have different potencies and affinities for hepatic functions. S: A COMPOUND is a reactive mobile objects carrying identification information. It maps to small fractions of a xenobiotic dose. When a COMPOUND generates a response, there is a ZoRLA counterpart to potency.

- A: A standard measure hepatic removal of a xenobiotic is intrinsic clearance $\left(\mathrm{CL}_{\text {int }}\right)$, the clearance rate absent blood flow. It depends in part on the relative amounts of enzymes and transporters expressed by each hepatocyte. S: The ZoRLA counterpart to $\mathrm{CL}_{\text {int }}$ is CLEARANCE effort. Each $\mathrm{SS}$ can change its CLEARANCE effort upon exposure to COMPOUNDS.

- A: Hepatocytes often exhibit location dependent expression of enzymes and transporters. S: SSs can exhibit location dependent CLEARANCE effort.

- A: Zonation of metabolic clearance occurs at both the cell and lobule level, implicating cell-cell cooperation at some level. It changes adaptively. S: Individual SSs can improve efficiency by adapting their CLEARANCE effort to changing COMPOUND exposures and to actions of other SS. 


\section{Sheikh-Bahaei and Hunt}

- A: Xenobiotic dosing typically first induces pericentral P450 isozymes. With increasing dose there is increasing induction in the periportal direction. S: A ZoRLA should be capable of producing patterns of CLEARANCE effort that mimic those observed in vivo over variety of experimental conditions.

- A: Compound dosing can cause zonal patterns of cell damage. S: It is straightforward to implement a ZoRLA counterpart of cell damage. When implemented it exhibits different zonal patterns.

\subsection{The Synthetic Method of Modeling and Simulation}

The method used is a relatively new experimental approach to discover and challenge plausible, biomimetic mechanisms. To gain insight into plausible generative mechanisms that may be responsible for zonation phenomena when uncertainty is large and detailed data are limited, the method involves building extant working mechanisms that exhibit some of those same phenomena, and thus may be biomimetic. The approach is based on the scientific principle (Darden, 2002) illustrated in Fig. 1A. When two systems, hepatic lobules of laboratory rats and a ZoRLA are composed of interacting components for which similarities can be established at some level of abstraction (mappings 1 in Fig. 1A), and the two systems exhibit several measurable, phenotypic attributes (mappings 3), for which some degree of similarity exists, then there may also be similarities in the generative mechanisms responsible for those attributes (mappings 2). We cannot yet build hierarchical mechanisms out of biochemicals. However, as described herein and recently reviewed (An et al., 2009; Fisher and Henzinger, 2007; Grimm et al., 2005; Hunt et al., 2009), we can build extant biomimetic mechanisms using object and agent-oriented software tools. In so doing, as Fig. 1 illustrates, we are not following the traditional, inductive approach of modeling the data. Nor are we describing mathematically the behaviors of a hypothetical, conceptual mechanism. Because the MECHANISMS are intended to be analogous to referent biological mechanisms, we refer to the in silico system as an analogue. To emphasize their concrete, constructive nature, we call them synthetic analogues. The approach provides an important, scientific and experimental means to explore and test in silico plausible, biomimetic mechanistic hypotheses, when it would be too difficult, too expensive, infeasible, or unethical to do so in animals or in vitro.

\subsection{ZoRLA Design Considerations}

In related work, we built liver analogues to challenge mechanistic hypotheses and improve insight into plausible micro-mechanistic details of xenobiotic clearance (Hunt et al., 2006; Yan et al., 2008a,b), drug interactions (Lam and Hunt, 2009), diseased-caused differences in spatiotemporal micro-mechanisms influencing hepatic drug disposition (Park et al., 2009), and heterogeneities in intralobular enzyme induction (Ropella et al., 2008). At the start of this project, we drew on these methods and their validated components to instantiate and experiment on lobular analogues targeting subsets of the above attributes.

All ZoRLA system components and processes are discrete. Time advances discretely by simulation steps. Each simulation step, every component updates its state based on changes since the last update opportunity. Each SS is an agent. SSs can be connected together in different ways. Within a simulation step, SS components interact with mobile COMPOUNDS percolating through a sequence of $20 \mathrm{SS}$. The process maps to absorbed xenobiotics percolating through sinusoids and interacting (or not) with spaces and cells as described in (Park et al., 2009; Yan et al., 2008a). Most events are stochastic. During a simulation step, when an event opportunity for a given component occurs, there is a parameter-specified probability for each, prespecified outcome.

A specific ZoRLA instantiates a mechanistic hypothesis (Fisher and Henzinger, 2007; Hunt et al., 2009): this component arrangement and operating principles will, upon execution, produce phenomena similar to targeted attributes. Execution followed by comparison of results to referent data tests the hypothesis. The process is directly analogous to constructing and then experimenting on a wet-lab model to test hypotheses. We adhered to a parsimony guideline and strove to keep ZoRLA components as simple as feasible, while achieving the targeted attributes. 


\section{Sheikh-Bahaei and Hunt}

\subsection{SS Intrinsic Clearance}

Primary targeted attributes include intralobular changes in compound clearance and levels of P450 isozymes (or their mRNA) responsible for that clearance. We needed an SS counterpart to intrinsic clearance. We can see from Fig. 2B that some of the compound entering an SS can exit without entering hepatocytes. A COMPOUND that is detected by an SS maps to compound entering hepatocytes. A COMPOUND that was undetected bypassed that SS. We specified a bypass probability (pBypass) for each SS that could be adjusted for each COMPOUND. A COMPOUND maps to a small fraction of the xenobiotic dose used in a wet-lab experiment. A COMPOUND that does not bypass is detected; that maps to compound entering cells. We specified that if detected, the COMPOUND would be cleared with probability $p$; that maps to the intrinsic clearance by hepatocytes within a corresponding sinusoidal segment for the time interval to which a simulation step maps. We define $p_{i, t}$ to be the CLEARANCE effort of $\operatorname{SS}_{i}(i=1,2, \ldots, 20)$ during simulation step $t$. Within hepatocytes, an increase in intrinsic clearance typically correlates with an increase in P450 isozymes. For this report, we limit attention to those cases. That increase comes at a cost to the cell. In SS, the cost of a COMPOUND clearance event is $k_{c}$. Given the many functions carried out by hepatocytes, we assumed that hepatocytes have an evolution imposed, genetic mandate to avoid unnecessary costs.

\subsection{The Linear ZoRLA System}

The ZoRLA in Fig. 2C is composed of 20 SS embedded within an extrahepatic space. The system maps abstractly to a portion of a hepatic lobule. SS mechanisms are described below. An SS has no knowledge of any other SS. There are two types of mobile objects: XENOBIOTICS and RESPONSE SIGNALS (RSIGNALS). A source container (not shown) for each is located just prior to $\mathrm{SS}_{1}$. Dosing can be delayed.

Figure 2D and E provide a view of the abstract mechanism within each SS agent. In a rat lobule, a compound entering a sinusoid segment can exit into the next segment without encountering hepatocytes. The same is true for an SS: a XENOBIOTIC that enters an SS can exit without being detected by (seen by) its CLEARANCE mechanism. The probability of bypassing an SS is specified by the parameter pBypass. A XENOBIOTIC will be detected with a probability of 1 - pBypass. Once detected, two events occur. First, the XENOBIOTIC either is or is not CLEARED. The primary requirement of the abstract SS CLEARANCE mechanism is that it be consistent with known hepatocyte details. The $\mathrm{SS}_{i}$ counterpart to intrinsic clearance is the probability $p_{i}$ that a detected XENOBIOTIC will be CLEARED, and that value is under $\mathrm{SS}_{i}$ control. Because each SS is quasi-autonomous, each can act independently. Increasing $p_{i}$ can map to induction of metabolizing enzymes and/or induction of uptake or efflux (to bile) transporters.

The second event is generation of a R-SIGNAL. The R-SIGNAL then exits that SS and enters each subsequent SS; in each it is detected (Fig. 2E). A fraction (in this study, 90\%) of R-SIGNALS that exits the ZoRLA is destroyed in the extrahepatic space. Those not destroyed return to the ZoRLA (maps to factors contained in returning portal vein blood) where they can again be detected. The XENOBIOTIC is a TOXIN if potency $\left(k_{t}\right) \geq 1$. Potency specifies the number of R-SIGNAL released by each SS that detects the TOXIN. For each R-SIGNAL detected, there is a cost, $k_{a}$ (Fig. 2E)

\subsection{Signals and Methods Needed and Used by SS}

Gebhardt argued that two classes of signals are necessary and essential for creating zonation (Gebhardt, 1992). We specified that R-SIGNALS be connected to COMPOUND, dose, and potency. We identified three options: 1) a COMPOUND or its METABOLITE is the R-SIGNAL; 2) a COMPOUND or its METABOLITE initiates a response within an SS and that response (or an object generated by that response) functions as a RSIGNAL that is also detectable subsequently by other SS; and 3) a COMPOUND or its METABOLITE that escapes HEPATIC clearance initiates an EXTRAHEPATIC response, and that response (or an object generated by that response) is an R-SIGNAL that is SS detectable. In this work, we focused on option two. Option two may map to a xenobiotic that causes hepatic tissue toxicity. The algorithm in Fig. $2 \mathrm{E}$ can be modified to give similar CLEARANCE effort changes for the third option, as well. For simplicity, we specified that a blood-born SIGNAL gradient, which is unrelated to xenobiotic, map to something external that is 


\section{Sheikh-Bahaei and Hunt}

carried to the liver by blood. It could, for example, map to $\mathrm{O}_{2}$ levels. Again, for simplicity, we specified that its input rate be constant, and we specified that a fixed linear P-to-P SIGNAL gradient exists, decreasing with increasing $i$, and that the value $g_{i}$ at each $\mathrm{SS}_{i}$ is constant for the duration of the simulation experiment.

\subsection{Enabling SS to Adapt CLEARANCE Effort in Response to Dosing with Different COMPOUNDS}

Christoffels et al. (1999) and others have posited that zonation is linked to the adaptive ability of the liver and hepatocytes specifically. The SS in Fig. 2E are very simple agents. Each SS has three actions: detect gradient SIGNALS, detect R-SIGNALS, and clear COMPOUNDS. We conjectured that if a cost were associated with each action, then when given a simple learning algorithm, an SS could use it to avoid increasing costs, and that would result in location dependent CLEARANCE effort. Consequently, a mandate of each SS was to alter CLEARANCE effort to avoid rising costs. We specified that the cost of clearing one COMPOUND is $k_{c}$. We conjectured that if a xenobiotic disrupts hepatocyte function or causes hepatocyte damage, the consequences of such an event could cause hepatocytes to alter their behaviors, and so doing would come at a cost to the hepatocytes. Generating and detecting R-SIGNALS maps to those events. We specified that the cost of detecting one R-SIGNAL is $k_{a}$. Increasing $p$ increases costs. Costs are lowest when an SS chooses $p=0$. However, for the case where $k_{t} \geq 1$ and $p=0$, TOXINS could be detected by each SS causing release of many R-SIGNALS and that would be costly to each SS. With a direct relation between TOXIN potency and the number of R-SIGNALS produced and detected, it is clear that P-to-P patterns of CLEARANCE effort would change as COMPOUND dose and potency were changed. We explored those changes. Based on recent observations about simulated hepatocyte learning (Sheikh-Bahaei et al., 2009), we anticipated that, given a simple learning mechanism (an algorithm in this case), an SS at the end of simulation step $t-1$ could find a new $p_{t}$ that would be expected to lower costs during subsequent simulation steps.

The SS mechanism in Fig. 2E provides the above capabilities and is simple enough so that there may be one or more yet to be identified hepatocyte counterparts. The figure shows an SS with a quasiautonomous subsystem, the CLEARANCE Management Module. It maps to all hepatocyte resources and subsystems associated with xenobiotic clearance. The CLEARANCE Management Module has one mandate: adjust $p$ up or down or keep it the same to lower future costs. A function in form of a Boltzmann distribution is provided that adjusts $p_{i, t}$.

$$
p_{i, t}=1 /\left(1+\exp \left[\left(g_{i}-Q_{i, t}\right) / h\right]\right)
$$

$Q_{i, t}$ is based on the well-known $Q$-learning algorithm (Watkins and Dayan, 1992). The value of $Q$ used by $\mathrm{SS}_{i}$ during simulation step $t$ is an estimate of its long-term, discounted cost of continuing to use its current CLEARANCE effort, $p_{t}$. At the start of each simulation step, $p_{t}$ is calculated using the $Q_{t}$ value carriedforward from the previous $(t-1)$ step (Eq. 1). At the end of the current step, $Q_{i, t}$ is updated using Eq. 2. $Q_{t+1}$ is then carried forward for use in the next step. $h$ is a constant that modulates the $g-\mathrm{Q}$ difference. When $h$ is very large, $p_{i, t}$ is constant at 0.5 independent of $\mathrm{Q}$ and $g$. When $h$ is very small, $p_{i, t}$ essentially takes one of two location dependent values.

$$
Q_{i, t+1}=(1-\alpha) Q_{i, t}-\alpha\left(k_{a}+k_{c}\right)
$$

$\alpha$ is a constant in $[0,1]$ that controls the ClEARANCE Management Module's learning rate. When a COMPOUND has been cleared, there is a cost to that SS: $k_{c}=1$, else $k_{c}=0$. When an R-SIGNAL is detected, there is also a cost to that SS: $k_{a}=1$, else $k_{a}=0$. The term $\left(k_{a}+k_{c}\right)$ represents the cost to each SS for the actions taken during a simulation step. At the end of each step, each SS updated its $Q$ value. As stated above, $g_{i}$ is the value of the P-to-P SIGNAL gradient used by SS$i$; for agent $i$ in this study, $g_{i}=\gamma(21-i) / 20$. 


\section{Sheikh-Bahaei and Hunt}

\section{RESULTS}

\subsection{Three Factors were Important in Determining Zonation Patterns During ZoRLA Experiments}

The first factor was potency. Operation of the Fig. 2E learning mechanism led to biomimetic patterns of functional zonation when used by 20 SSs connected in series (Fig. 2C). The R-SIGNAL generation and detection mechanism was as illustrated in Fig. 2D. When the duration of repetitive exposure to the same COMPOUND was long enough, the patterns produced always stabilized. The four sets of results in Fig. 3 used the same parameterization, but the three COMPOUNDS had different potencies $\left(k_{t}\right.$ values). Prior to dosing, each SS's $Q$ value was initialized to zero. There are two measures of zonation. One is the value of $p_{A V G}$ (it maps to intrinsic clearance), which is the probability that an SS will clear a COMPOUND if it is detected, averaged over the past $N_{\text {avg }}$ simulation steps; for these studies, we used $N_{\text {avg }}=100$. The second is COMPOUND Elimination Count; it is the number of COMPOUNDS CLEARED by an SS. COMPOUND Elimination Count maps to hepatocyte exposure. When $k_{t}=0$ (and pBypass is large-0.99 in Fig. 3), only perivenous SSs expended CLEARANCE effort. As $k_{t}$ increased, $p_{A V G}$ increased in the periportal direction. Note that when potency changed, each SS's $Q$ value also changed, and that caused its $p_{A V G}$ and COMPOUND Elimination Count to change. We can reasonably infer that hepatocyte exposure is directly correlated with total amount of compound cleared by that hepatocyte. Consequently, if the COMPOUND is HEPATOTOXIC, then we can expect measures of HEPATOTOXICITY to correlate with COMPOUND Elimination Count. For the experiments in Fig. 3, the change in COMPOUND Elimination Count with increasing dose followed a trend similar to that for $p_{A V G}$.

The second factor was pBypass. Having pBypass $=0.99$ can map to compound that is highly bound to one or more blood components or to a compound with a small partition coefficient. For the results in Fig. 4, we decreased pBypass to 0.9, while keeping all other ZoRLA parameter values unchanged. The $p_{A V G}$ pattern as $k_{t}$ increased was similar to that in Fig. 3. However, unlike the trend in Fig. 3, the peak COMPOUND Elimination Count moved toward the periportal region with increasing potency.

The third factor was dose. Figure 5 shows consequences of simulated dose changes for the COMPOUND in Figure 3 having $k_{t}=1$. Increased simulated dose produced changes in $p_{A V G}$ and COMPOUND Elimination Count that exhibit trends similar those in Fig. 3 where potency increased: a large dose of a low potency COMPOUND produced results similar to those from a small dose of a high potency compound, a result that is biomimetic.

Shown in Figure 3 are zonation patterns across 20 SSs following constant input $(2,000$ simulation steps) of each of four COMPOUNDS having different potencies. Each SS used the learning-enabled, clearance mechanism in Fig. 2E. Left: the average CLEARANCE efforts, $p_{\mathrm{AVG}}$ (blue bars), as well as COMPOUND Elimination Count (red bars) for each SS are shown. $p_{\mathrm{AVG}}$ is the probability that an SS will clear a COMPOUND if it is detected, averaged over the past $\mathrm{N}_{\mathrm{avg}}=100$ simulation steps. COMPOUND Eli-

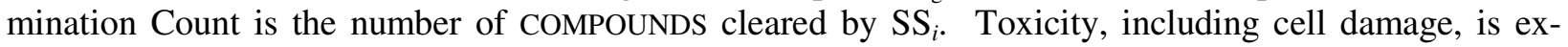
pected to correlate directly with that value. Right: corresponding $g$ (gradient value at each SS) and $Q$ values are also shown for each potency. $\mathrm{N}_{\text {agents }}$ : number of SS; $\mathrm{N}_{\text {step }}$ : maximum number of simulation

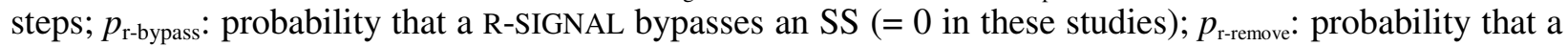
R-SIGNAL object is removed by an encountered SS ( $=0$ in these studies); $k_{t}$ : number of R-SIGNAL objects generated by each SS upon TOXIN detection; tox_speed_ratio: the ratio R-SIGNAL/TOXIN speed; a_recycle_fract: fraction of R-SIGNALS that first exit and then reenter a ZoRLA in a subsequent step. 

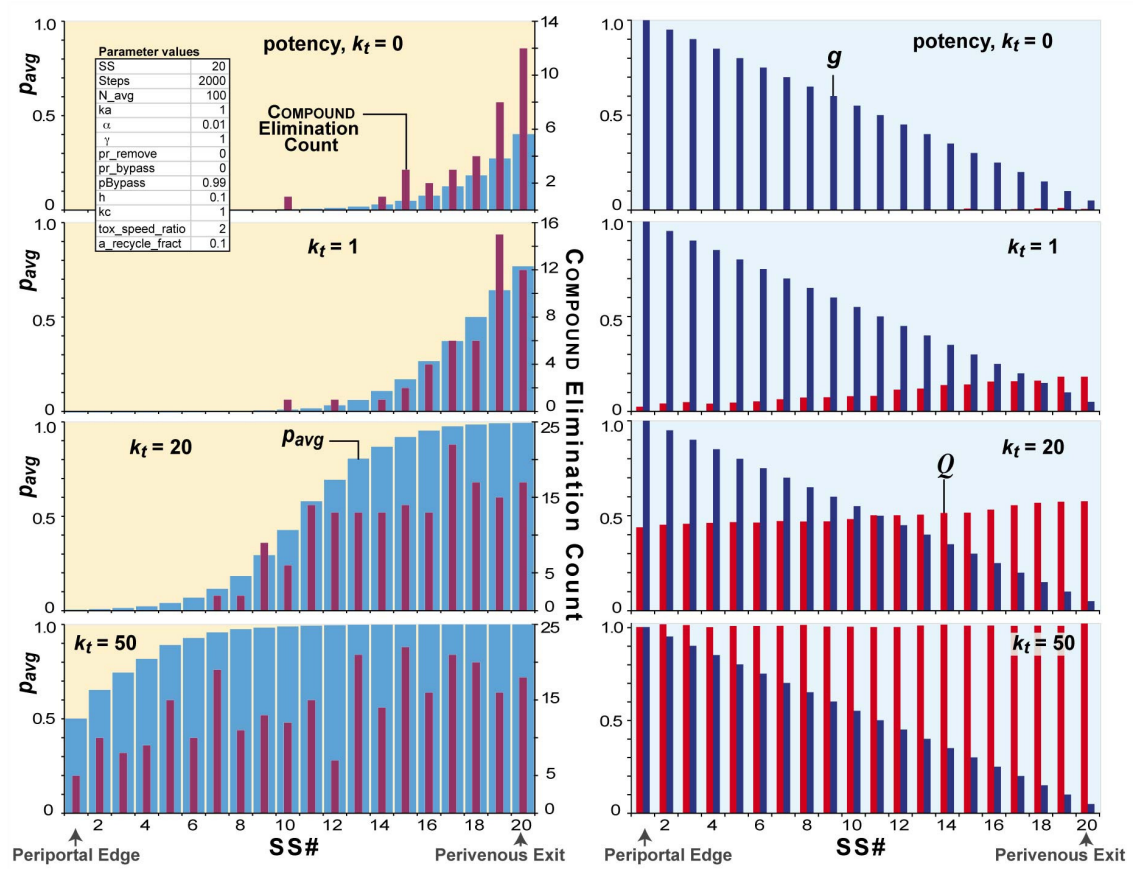

Figure 3: Examples of ZoRLA generated patterns.

Shown in Figure 4 are zonation patterns across 20 SSs following constant input (2,000 simulation steps) of each of four COMPOUNDS having different potencies. The experiment is the same as that in Fig. 3 , except that pBypass was decreased from 0.99 to 0.9. So doing caused peak CoMPOUND Elimination Count values to shift from perivenous to periportal as potency increases.
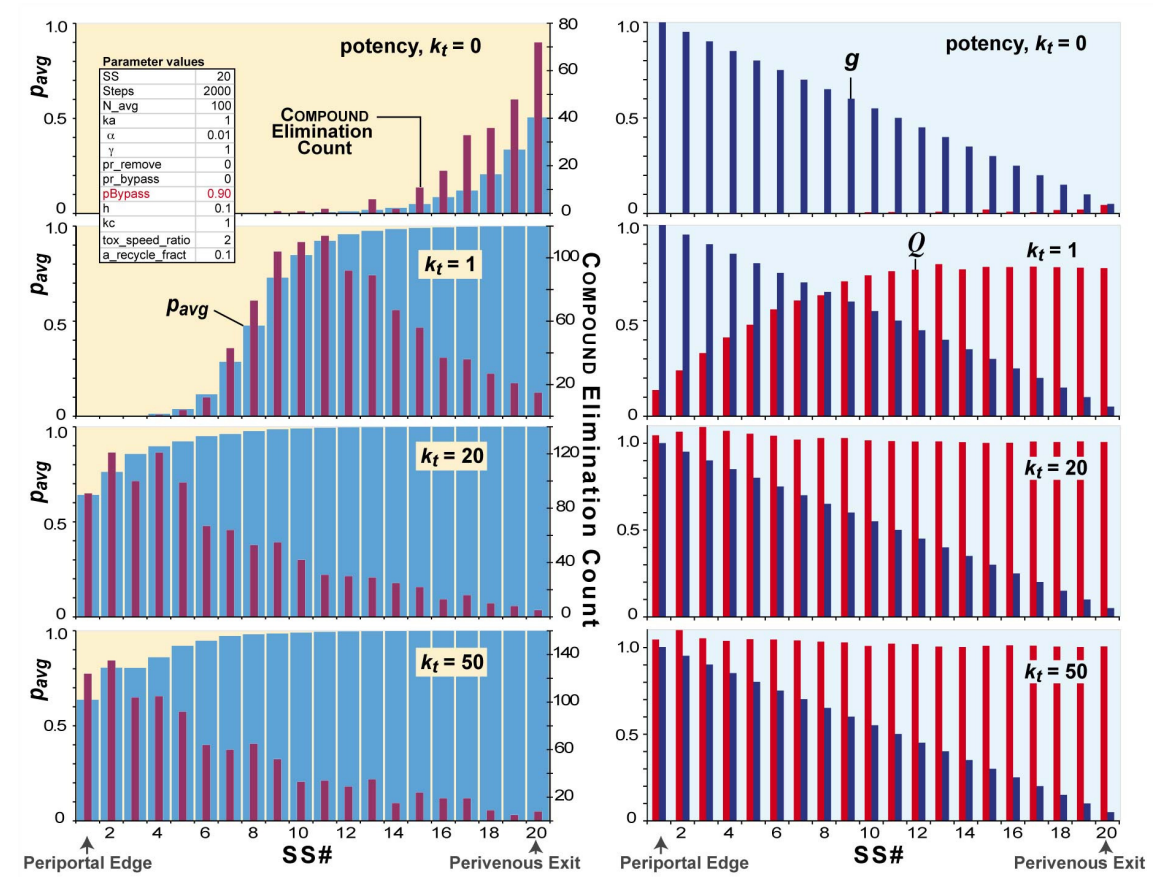

Figure 4: Examples of ZoRLA generated patterns. 


\section{DISCUSSION}

\subsection{Plausible Mappings of SS Mechanisms to Hepatic Counterparts}

When designing a synthetic analogue, there is a strong inclination to insert counterparts to specific biological features, such as including a component that maps directly to the factors within signaling pathways, simply because current evidence indicates that the component plays some role in generating the targeted phenomena. Doing so prematurely is a mistake when we are not yet confident of the component's putative role in causing the phenomena. So doing forces establishing groundings to other components at a specific level of granularity that may or may not be warranted for the attributes targeted coupled with the current levels of knowledge, ignorance, and uncertainty. The components in Figs. 2 are more abstract and coarse-grained than signaling pathways. They were made only as complicated and specific as needed to achieve the patterns and attributes targeted. They can be made more fine-grained and specific iteratively, as the set of attributes targeted expands. Once a degree of validation has been achieved for coarsegrained components, their behaviors during simulation can be used for cross-model validation during refinement to more fine-grained (greater mechanistic detail) counterparts.
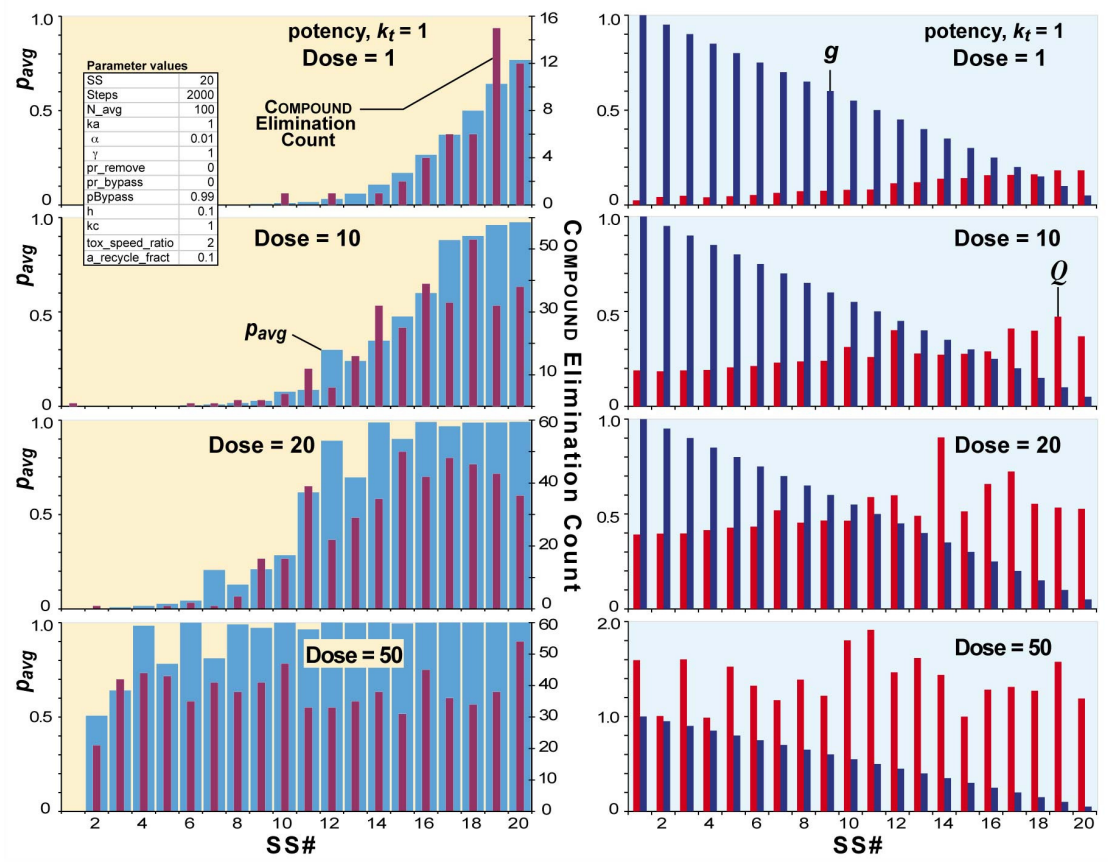

Figure 5: Examples of ZoRLA generated patterns.

The ZoRLA is the same as specified in Fig. 3. Shown are zonation patterns across 20 SSs following constant input of different simulated doses of the COMPOUND in Fig. 3 having potency, $k_{t}=1$. So doing caused COMPOUND Elimination Count values to increase in the periportal direction as dose increased.

\subsection{Inverse Maps from Phenomena to Generators}

The exploration of an inverse map from phenomena (patterns of zonation) to generators requires one to hypothesize and then build generators that may cause indistinguishable phenomena. The question posed is this: given phenomena such as intralobular zonation, what plausible generators might cause their emer- 


\section{Sheikh-Bahaei and Hunt}

gence over time? However, a generator-phenomenon map is not one-to-one. Many generator compositions (mechanisms) can produce phenomena, which when measured are indistinguishable. Nevertheless, the first step is to find and validate one, which is what we have done here. So doing opens the door to discovering others. When we find a hypothetical generator in the form of an extant biomimetic mechanism, we do not yet have new biological knowledge, but we do have a concrete instance of a strictly defined, plausible and observable mechanism within a system suitable for experimentation, as called for by Christoffels et al. (1999), whereas before we only had unchallenged concepts. In the absence of other concrete, competing theories, that system and its mechanism can stand as a plausible explanation of the phenomena's cause until falsified by evidence. A systematic, scientific study of any inverse map (phenomenon-to-generator) can only be done with concretizable hypotheses, either biological or in silico. It cannot be done with hypotheses that remain conceptual (Hunt et al., 2009).

As this $M \& S$ method matures, we anticipate that the preferred approach will be to identify several somewhat different yet still plausible generator models and refine them in parallel against an expanding set of targeted attributes. We can expect that modest, selective expansions in the set of targeted attributes will eliminate some generator concepts but allow others to advance following refinement.

\subsection{Objective Achieved}

In Methods, we specified that R-SIGNALS are COMPOUND dependent, and listed three mechanistic options: 1) a COMPOUND or its METABOLITE functions as a R-SIGNAL; 2) a COMPOUND or its METABOLITE initiates a response within an SS and that response (or an object generated by that response) functions as a RSIGNAL that is also detectable subsequently by other SS; and 3) a COMPOUND or its METABOLITE that escapes clearance initiates an EXTRAHEPATIC response, and that response (or an object generated by that response) is an R-SIGNAL that is SS detectable. Exploration of the third option is needed. Exploratory simulations (not presented) showed that zonation does occur when using COMPOUNDS as R-SIGNALS for single-pass dosing experiments. However, further exploration demonstrated that the first option could not achieve the attribute targeting adaptability because the R-SIGNALS generated stay within each SS and so cannot influence other SSs. It seems likely that there are several mechanisms by which zonation of hepatic cellular properties could occur. The approach used herein can be extended to discover and investigate those options.

Based on the evidence presented, we suggest that hepatic counterparts to the abstract ZoRLA mechanism, along with the zonation patterns produced, exist in rats when treated with some xenobiotics. The implication of these ZoRLA experiments is that hepatocytes do learn from experience and can cooperate to remove xenobiotics, including some that produce significant response or toxicity.

The results also demonstrate a new scientific method to experimentally explore and challenge mechanistic hypotheses about the causal nature of toxicologic and pharmacologic phenomena.

\section{ACKNOWLEDGMENTS}

We thank members of the UCSF BioSystems Group for helpful suggestions and discussions. The work was supported in part by a Computational and Systems Biology Fellowship (SSB) provided by the CDH Research Foundation (CDHRP-08-0044). An expanded version of this report is now available: SheikhBahaei S, Maher JJ, Hunt CA. (2010) Computational Experiments Reveal Plausible Mechanisms for Changing Patterns of Hepatic Zonation of Xenobiotic Clearance and Hepatotoxicity. J. Theor. Biol., DOI: 10.1016/j.jtbi.2010.06.01.

\section{REFERENCES}

An, G., Q. Mi, J. Dutta-Moscato, and Y. Vodovotz. 2009. Agent-based models in translational systems biology. Wiley Interdiscip. Rev. Syst. Biol. Med. 1:159-171. 


\section{Sheikh-Bahaei and Hunt}

Benhamouche, S., T. Decaens, C. Perret, and S. Colnot. 2006. Wnt/ $\beta$-catenin pathway and liver metabolic zonation: a new player for an old concept. Med Sci 22:904-906.

Braeuning, A. 2009. Regulation of Cytochrome P450 Expression by Ras-and-Catenin-Dependent Signaling. Curr. Drug Metab. 10, 138-158.

Burke, Z.D., K.R. Reed, T.J. Phesse, O.J. Sansom, A.R. Clarke, and D. Tosh. 2009. Liver Zonation Occurs Through a _-Catenin-Dependent, c-Myc-Independent Mechanism. Gastroenterology 136:23162324.

Camp, J.P., and A.T. Capitano. 2007. Induction of zone-like liver function gradients in HepG2 cells by varying culture medium height. Biotechnol. Prog. 23:1485-1491.

Christoffels, V.M., Sassi, H., J.M. Ruijter, A.F. Moorman, and T.L. Grange. 1999. A mechanistic model for the development and maintenance of portocentral gradients in gene expression in the liver. Hepatology 29:1180-1192.

Darden, L. 2002. Strategies for discovering mechanisms: Schema instantiation, modular subassembly, forward/backward chaining. Phil. Sci. 69:354-365.

Fisher, J., and T.A. Henzinger. 2007. Executable cell biology. Nat. Biotechnol. 25:1239-1250.

Gebhardt, R. 1992. Metabolic zonation of the liver: regulation and implications for liver function. Pharmacol. Ther. 53:275-354.

Grimm, V., E. Revilla, U. Berger, F. Jeltsch, W.M. Mooij, S.F. Railsback, H.H. Thulke, J. Weiner, T. Wiegand, and D.L. DeAngelis. 2005. Pattern-oriented modeling of agent-based complex systems: lessons from ecology. Science 310:987-991.

Hailfinger S., M. Jaworski, A. Braeuning, A. Buchmann, and M. Schwarz 2006 Zonal gene expression in murine liver: Lessons from tumors. Hepatology 43:407-14.

Hunt, C.A., G.E.P. Ropella, T.N. Lam, J. Tang, S.H.J. Kim, J.A. Engelberg, and S. Sheikh-Bahaei. 2009. At the Biological Modeling and Simulation Frontier. Pharm. Res. 26:2369-2400.

Hunt, C.A., G.E.P. Ropella, L. Yan, D.Y. Hung, and M.S. Roberts. 2006. Physiologically based synthetic models of hepatic disposition. Journal of pharmacokinetics and pharmacodynamics 33:737-772.

Jungermann, K. 1995. Zonation of metabolism and gene expression in liver. Histochem. Cell Biol. 103, 81-91.

Jungermann, K., and T. Kietzmann. 2000. Oxygen: modulator of metabolic zonation and disease of the liver. Hepatology 31:255-260.

Lam, T.N., and C.A. Hunt. 2009. Discovering plausible mechanistic details of hepatic drug interactions. Drug Metab. Disposition 37:237-246.

Lindros, K.0. 1997. Zonation of cytochrome P450 expression, drug metabolism and toxicity in liver. Gen. Pharmacol. 28:191-196.

Oinonen, T., and K.O. Lindros. 1998. Zonation of hepatic cytochrome P-450 expression and regulation. Biochem. J. 329:17-35.

Park, S., G.E.P. Ropella, S.H.J. Kim, M.S. Roberts, and C.A. Hunt. 2009. Computational strategies unravel and trace how liver disease changes hepatic drug disposition. J. Pharmacol. Exp. Ther. 328:294305.

Ropella, G.E.P., Park, S., and C.A. Hunt. 2008. Evaluating an hepatic enzyme induction mechanism through coarse-and fine-grained measurements of an in silico liver. Complexity 14:28-34.

Sekine, S., B.Y.A. Lan, M. Bedolli, S. Feng, and M. Hebrok. 2006. Liver-specific loss of $\beta$-catenin blocks glutamine synthesis pathway activity and cytochrome p450 expression in mice. Hepatology 43:817-825.

Sheikh-Bahaei, S., S.H.J. Kim, and S. Sheikhbahahaei, C.A. Hunt. 2009. Understanding the Role of Liver Zonation in Toxin Elimination. Int. J. Intell. Control Syst. 14:33-40.

Watkins, C. J. C. H., and P. Dayan. 1992. Technical Note: Q-Learning. Mach. Learn. 8:279-292.

Yan, L., G.E.P. Ropella, S. Park, M.S. Roberts, and C.A. Hunt. 2008. Modeling and simulation of hepatic drug disposition using a physiologically based, multi-agent in silico liver. Pharm. Res. 25:1023-1036.

Yan, L., S. Sheihk-Bahaei, S. Park, G.E.P. Ropella, and C.A. Hunt. 2008. Predictions of hepatic disposition properties using a mechanistically realistic, physiologically based model. Drug Metab. Disposition 36:759-768. 


\section{AUTHOR BIOGRAPHIES}

SHAHAB SHEIKH-BAHAEI received B.S. degree in Electrical Engineering in 1999 from Isfahan University of Technology, Isfahan, Iran; and M.S. degree in Control Engineering in 2003 from University of New Mexico, Albuquerque, USA. He received his Ph.D. in Bioengineering jointly from University of California, San Francisco and Berkeley in June 2010. His areas of interest include modeling and simulation, multi-agent systems, self-organizing systems, complex systems, networks and artificial intelligence. His email address is <shahabealum. berkeley.edu>.

C. ANTHONY HUNT is Professor of Bioengineering and Therapeutic Sciences at the University of California, San Francisco. He earned B.S. degrees in Chemistry and Applied Biology, served as an aviator in the U.S. Navy, earned a Ph.D. in Pharmaceutical Chemistry, and worked as discovery scientist before joining the UCSF faculty. He directs the BioSystems Group (biosystems.ucsf.edu). Its primary goal is to use of advanced modeling and simulation methods $(\mathrm{M} \& \mathrm{~S})$ to develop, instantiate, challenge, and improve hierarchical theories of drug-organism-environment interactions and consequences. The theories specify plausible causal linkages between molecular level events within higher-level mechanisms to aspects of pharmacological and toxicological phenotype in vitro and in vivo. Prof. Hunt is a fellow of the American Association for the Advancement of Science and of the American Association of Pharmaceutical Scientists. His email address is $<$ a. hunteucsf . edu $>$. 